\title{
Gastric Mixed Adenoneuroendocrine Carcinoma with Revised Diagnosis after Retrospective Pathologic Review
}

\author{
Bo Seok Jeong', Hye Won Hwang' ${ }^{2}$, Jong Won Kim³ , In Gyu Hwang ${ }^{1}$, Jae Yong Park ${ }^{1}$ \\ Departments of Internal Medicine ${ }^{1}$, Pathology ${ }^{2}$, and Surgery ${ }^{3}$, Chung-Ang University College of Medicine, Seoul, Korea
}

\begin{abstract}
Gastric mixed adenoneuroendocrine carcinoma (MANEC) is an entity defined by World Health Organization in 2010, which is composed of adenocarcinoma and neuroendocrine neoplasm. This is a very rare type of tumor in the stomach, which is estimated to be one to two per one million people per year on gastrointestinal track. In general, as MANEC often shows a poor prognosis, early diagnosis of the disease is important for achieving a favorable outcome. However, due to its rarity and histological heterogeneity, it is hard to suspect this disease initially, and sometimes this tumor is misdiagnosed as adenocarcinoma. The present case was a 76-year-old man who was initially diagnosed with gastric tubular adenocarcinoma without distant metastasis. Only 1 month after a curative surgery, multiple liver metastases were newly detected. Retrospective pathologic review resulted in a revised diagnosis as a gastric MANEC, and the patient underwent palliative systemic chemotherapy to achieve partial response. (Korean J Helicobacter Up Gastrointest Res 2021;21:343-348)
\end{abstract}

Key Words: Neoplasms, complex and mixed; Neuroendocrine tumors; Stomach neoplasms

\section{INTRODUCTION}

A gastrointestinal tumor in which neuroendocrine and exocrine components were both present was first reported by Cordier ${ }^{1}$ in 1924. Since then, there have been several case reports of this category of disease. In an atempt to define this disease and unify the terminology, The World Health Organization (WHO) announced the term "mixed adenocarcinoma-neuroendocrine carcinoma (MANEC)" for a mixed neoplasm with both neuroendocrine and exocrine component in 2010. Their definition includes for a mixed neoplasm with both neuroendocrine and exocrine component, where each component should represent at least 30\% of the lesion. This disease spectrum is now included under the category of "mixed neuroendocrine non-neuroendocrine neoplasm (MiNEN)", according to a recently updated WHO classification. ${ }^{2,3}$

Although the actual prevalence of MANEC is not precisely defined, it is estimated to be one to two per one million people per year on gastro-intestine. ${ }^{4}$ The optimal treatment strategy and prognosis of MANEC is largely un-

Received: September 26, 2021 Revised: October 31, 2021 Accepted: November 11, 2021 Corresponding author: Jae Yong Park

Department of Internal Medicine, Chung-Ang University College of Medicine, 84 Heukseok-ro, Dongjak-gu, Seoul 06974, Korea

Tel: +82-2-6299-3161, Fax: +82-2-6299-2064, E-mail: jay0park@cau.ac.kr known and still under discussion, due to the rarity of this disease.

Although early diagnosis and curative resection of localized disease is essential to achieve a better prognosis. Diagnosis of MANEC can often be difficult due to its very low prevalence and histological heterogeneity. In particular, gastric MANEC is frequently diagnosed initially as adenocarcinoma by endoscopic biopsy, leading to a delay in proper diagnosis. ${ }^{5}$ We hereby present a case of gastric tumor, initially diagnosed as adenocarcinoma, which was later revised as gastric MANEC by retrospective pathologic review after a clinical suspicion.

\section{CASE REPORT}

A 76-year-old man was referred from a local clinic due to suspected gastric cancer detected on a screening upper endoscopy. There were no specific findings in the blood test performed at the time of admission, only mild elevation of CEA was observed (Table 1). On esophagogastroduodenoscopy (EGD), a depressive lesion of about $3 \mathrm{~cm}$ was observed at the lesser curvature side of gastric high body (Fig. 1). Endoscopic biopsy revealed moderately differentiated adenocarcinoma on the histological examination.

Copyright $\odot 2021$ Korean College of Helicobacter and Upper Gastrointestinal Research

(a) The Korean Journal of Helicobacter and Upper Gastrointestinal Research is an Open-Access Journal. All articles are distributed under the terms of the Creative Commons Attribution Non-Commercial License (http:// creativecommons.org/licenses/by-nc/4.0) which permits unrestricted non-commercial use, distribution, and reproduction in any medium, provided the original work is properly cited. 
Table 1. Initial Blood Test Results of The Patient at the Time of Admission

\begin{tabular}{lcc}
\hline Blood test & Value & Reference range \\
\hline WBC $\left(10^{9} / \mathrm{L}\right)$ & 5.270 & $3.0-9.0$ \\
Hemoglobin $(\mathrm{g} / \mathrm{dL})$ & 12.7 & $13-17$ \\
Platelet $\left(10^{9} / \mathrm{L}\right)$ & 183 & $140-400$ \\
BUN $(\mathrm{mg} / \mathrm{dL})$ & 21 & $8-19$ \\
Creatinine $(\mathrm{mg} / \mathrm{dL})$ & 1.06 & $0.67-1.17$ \\
AST $(\mathrm{IU} / \mathrm{L})$ & 27 & $0-34$ \\
ALT $(\mathrm{IU} / \mathrm{L})$ & 32 & $0-40$ \\
Total bilirubin $(\mathrm{mg} / \mathrm{dL})$ & 0.5 & $0.3-1.3$ \\
LDH $(\mathrm{IU} / \mathrm{L})$ & 176 & $110-200$ \\
High sensitivity CRP $(\mathrm{mg} / \mathrm{L})$ & 1.24 & $0-1.0$ \\
CEA (ng/mL) & 5.50 & $0-4$ \\
CA $19-9(\mathrm{U} / \mathrm{mL})$ & 27.84 & $0-32$ \\
\hline
\end{tabular}

WBC, white blood cell; BUN, blood urea nitrogen; AST, aspartate aminotransferase; ALT, alanine aminotransferase; LDH, lactate dehydrogenase; CRP, C-reactive protein; CEA, carcinoembryonic antigen; CA, carbohydrate antigen.
As there was no evidence of distant metastasis on the subsequent staging workup including abdominopelvic CT (Fig. 2A), laparoscopy-assisted total gastrectomy with D2 lymphadenectomy was performed (Fig. 3). The lesion was poorly differentiated adenocarcinoma with a gross type of advanced gastric cancer Borrmann type III. It extended to the muscular propria and showed lymphovascular invasion but there was no metastasis in lymph node (pT2N0). Resection margin was free of tumor.

One month later, he complained of weight loss accompanied by dysphagia. As anastomotic stricture was observed on EGD, endoscopic balloon dilatation was performed. However, after the endoscopic procedure, he complained of chilling sense, and a chest CT was performed to exclude the possibility of perforation. Although
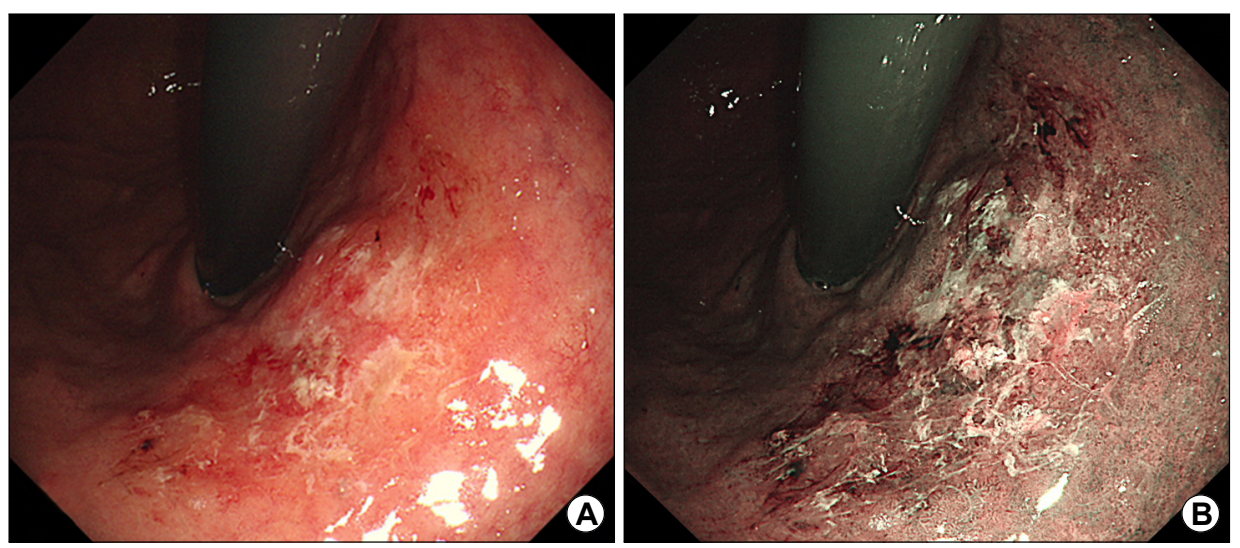

Fig. 1. Endoscopic finding of a malignant lesion. (A) Upper endoscopic examination shows a 3-cm sized ill-defined depressive lesion at the lesser curvature side of gastric high body. (B) The boundary of the lesion is more clearly visualized when observed in narrow band imaging mode.
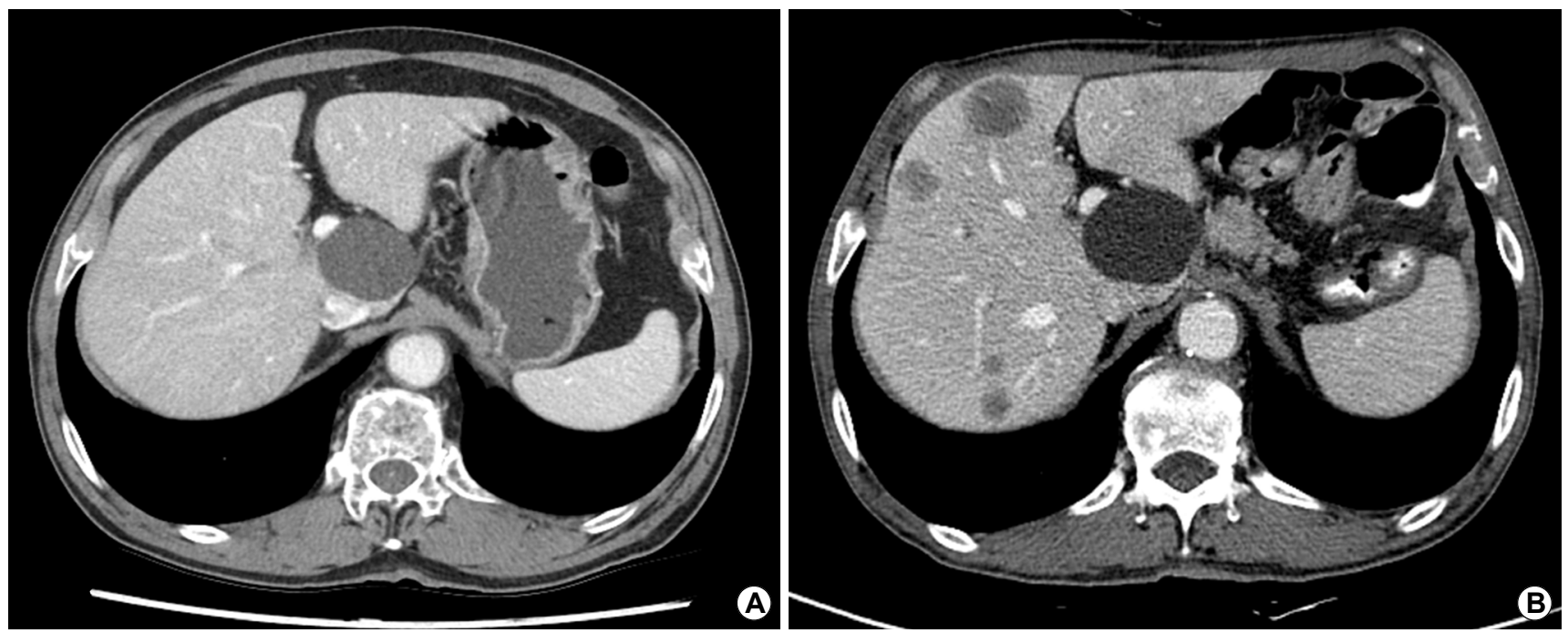

Fig. 2. Comparison of abdominopelvic CT images taken at the time of initial diagnosis and after surgery. (A) Image at the time of initial diagnosis. (B) Image showing newly developed multiple low attenuating masses in the liver, measuring up to $3.5 \mathrm{~cm}$. 
there was no evidence of perforation, nodular hepatic lesions were incidentally identified on the covered CT image.

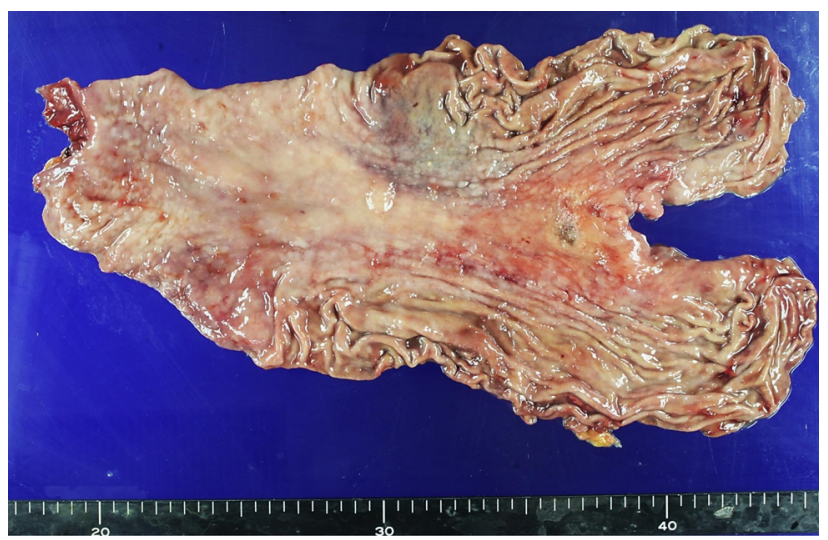

Fig. 3. Surgical specimen from the total gastrectomy with D2 lymphadenectomy, measuring $1.5 \times 1.0 \times 0.4 \mathrm{~cm}$, grossly classified as AGC Borrmann type III. AGC, advanced gastric cancer.
Subsequent abdominopelvic CT revealed newly detected multiple hepatic metastases (Fig. 2B), which was a very unusual finding in a patient who had undergone radical surgical resection with extensive lymph node dissection for stage IB gastric cancer.

With clinical suspicion, the surgical specimen was meticulously reviewed after a multidisciplinary team meeting. The pathologic review revealed a component of neuroendocrine differentiation in submucosa to muscular propria adjacent to the differentiated adenocarcinoma portion (Fig. 4). Subsequent immunohistochemical staining with chromogranin and synaptophysin both showed positive findings (Fig. 5A, B). In addition, the mitotic count was $>20 / 10$ high power fields and Ki-67 index was $>20 \%$ (Fig. 5C). Based on these findings, the diagnosis was revised as a gastric MANEC, composed of moderately differentiated
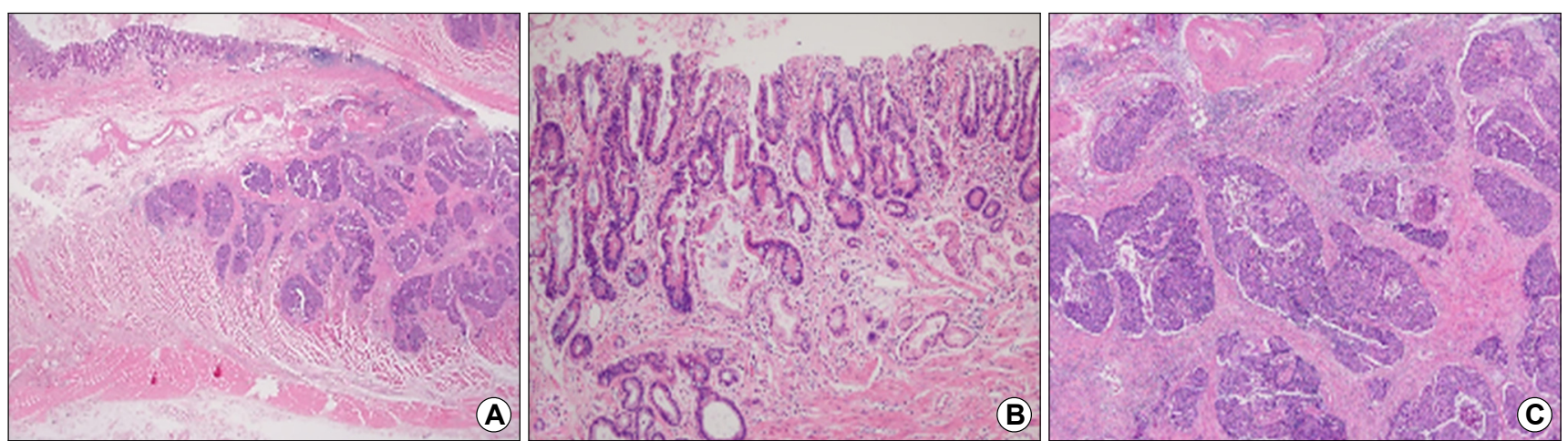

Fig. 4. Histologic features of the gastric tumor. (A) High grade MiNEN composed of adenocarcinoma (left upper) and poorly differentiated neuroendocrine carcinoma (right lower) (H\&E stain, $\times 12.5)$. (B) Moderately differentiated adenocarcinoma component is mainly confined to the mucosa layer (H\&E stain, $\times 100$ ). (C) Poorly differentiated neuroendocrine carcinoma is identified adjacent to the adenocarcinoma portion, invading the submucosa and muscularis propria (H\&E stain, $\times 40)$. MiNEN, mixed neuroendocrine non-neuroendocrine neoplasm.
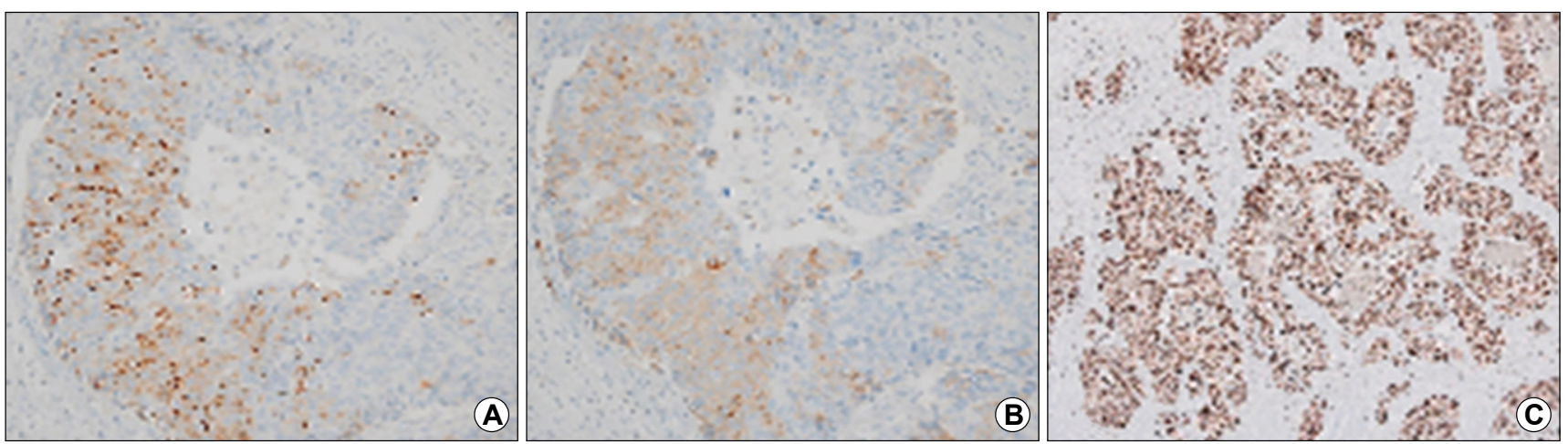

Fig. 5. The immunohistochemical staining results of the neuroendocrine component of the gastric tumor. (A) Focal positive cytoplasmic staining for synaptophysin (immunohistochemical staining, $\times 200$ ). (B) Focal positive cytoplasmic staining for chromogranin A (immunohistochemical staining, $\times 200$ ). (C) Ki-67 proliferation index is more than $50 \%$ (immunohistochemical staining, $\times 40$ ). 
adenocarcinoma and poorly differentiated neuroendocrine carcinoma (NEC).

The patient underwent 5-fluorouracil (5-FU), leucovorin and oxaliplatin chemotherapy (FOLFOX, 5-FU $1200 \mathrm{mg} / \mathrm{m}^{2}$, leucovorin $200 \mathrm{mg} / \mathrm{m}^{2}$, and oxaliplatin $85 \mathrm{mg} / \mathrm{m}^{2}$ ) at 2-week intervals. After the 9th cycle, progressive disease with increased hepatic metastases was confirmed on a follow-up CT. Liver biopsy was performed, and NEC component was predominantly observed along with adenocarcinoma. The chemotherapy regimen was then changed to paclitaxel $80 \mathrm{mg} / \mathrm{m}^{2}$ every 1 week and ramucirumab $8 \mathrm{mg} / \mathrm{kg}$ every 2 weeks. After three cycles of second line chemotherapy, the size of multiple hepatic metastases decreased. At this point, that is, about 9 months after the diagnosis of gastric MANEC, as the patient wanted to continue treatment near his residence, he was transferred to another hospital and follow-up was stopped at our hospital.

\section{DISCUSSION}

Ever since a gastrointestinal tumor in which neuroendocrine and exocrine component was co-existing was first reported by Cordier ${ }^{1}$ in 1924, several cases had been reported under different names until the WHO announced the term MANEC for this mixed neoplasm in 2010. ${ }^{3}$ This term refers to a neoplasm showing considerable histological heterogeneity, characterized by simultaneous presence of adenocarcinoma and neuroendocrine differentiation, each component representing at least 30\% of the tumor. ${ }^{6}$ However, as there were criticisms that the term does not convey the morphological and biological heterogeneity of this mixed neoplasm adequately, WHO recently proposed to use the term MiNEN to include histological variants other than adenocarcinoma and those with low-grade malignant components. Thereafter, MANEC now belongs to MiNEN in the 2019 WHO classification. ${ }^{2}$

Generally, MiNENs are highly aggressive neoplasms with poor prognosis. MiNEN can be classified into high, intermediate, and low-grade according to the metastatic potential of the two components. High-grade MiNEN refers to a mixed neoplasm composed of poorly-differentiated NEC and non-neuroendocrine carcinoma or adenoma ${ }^{7}$ as is our case. When well-differentiated neuroendocrine tumor (NET) is mixed with either non-neuroendocrine car- cinoma or adenoma, it is defined as intermediate-grade and low-grade disease, respectively. Previously, it was thought that the volume ratio of the two components would determine the clinical outcome of high grade MiNEN, but a recent retrospective analysis shows that the clinical course and prognosis of MANEC can vary depending on the degree of differentiation of the components, not the ratio of each component. ${ }^{7-9}$ In other words, any component with poor differentiation can become a subject of metastasis and mainly affect the patient's prognosis with MiNEN. In this manner, the NEC component is usually the most aggressive component in high-grade MiNEN. ${ }^{10}$

The diagnosis of MiNEN is usually determined by appearance in H\&E staining, immunohistochemical staining with markers for NETs such as synaptophysin, CD 56, and chromogranin, mitotic counts and Ki-67 index. ${ }^{11,12}$ However, caution is needed in the diagnosis of these mixed tumors for the following reasons. First, there are cases where neuroendocrine cells do not always respond to specific markers. ${ }^{13-15}$ Second, as can be seen in this case, the concordance between the pathological diagnosis before and after surgery for mixed neoplasm is low. ${ }^{13}$ Although several hypotheses have been suggested regarding the etiology of MiNEN, it has not yet been clearly elucidated, and but there are still controversies due to lack of data. ${ }^{12,15}$

Unlike pure gastric NETs, the treatment and follow-up strategy of gastric MiNEN has not been clearly established. In spite of limited data, localized disease should be treated with curative surgical resection whenever possible. Adjuvant or perioperative chemotherapy can be considered according to the grade of the disease. ${ }^{7}$ For a metastatic disease, although systemic chemotherapy is the mainstay of treatment, the optimal treatment regimen is not standardized based on a high evidence level. In our case, the patient achieved disease control with palliative chemotherapy for more than about 9 months.

For high-grade MiNENs, especially MANEC, the actual prevalence is not precisely defined, but is estimated to be $1 \sim 2$ cases per million people per year on gastro-intestine. ${ }^{4}$ Due to its scarcity, it has been mainly reported through case reports and case series in the stomach. About 20\% of all gastrointestinal MANECs are found in the stomach, most of which are diagnosed at an advanced stage. Due to the rarity of this diagnosis, the limited quality of published data 
and inconsistent terminology, the epidemiology, prognosis, and best treatment management of MiNEN are still under discussion. ${ }^{16-18}$ As there is chance for cure when curative resection is done for localized disease, early diagnosis is necessary for a better prognosis. However, in early stages, invasion is often limited to submucosa and diagnosed as tubular adenocarcinoma in endoscopic biopsy, which makes it difficult to accurately diagnose before surgery. ${ }^{12,19}$ Even after surgical resection, it is sometimes not easy to suspect MiNEN in the first place, as was in our case.

In previously reported cases of gastric MANEC, the location is distributed throughout the stomach, and the size varies from several millimeters to more than $10 \mathrm{~cm}$. Endoscopic morphologies are superficially raised or superficially depressed in most cases. Also clinically, metastases are more frequently observed in the liver and lymph nodes. ${ }^{5}$ However, these are not pathognomonic features of gastric MiNEN which distinguish it from gastric adenocarcinoma.

In the present case, the clinical course was very unusual for a stage IB gastric cancer without lymph node involvement, showing early distant metastasis after curative surgery. This raised a question to the initial diagnosis, and subsequent multidisciplinary approach and pathologic review resulted in a revised diagnosis as a gastric MiNEN. The poorly differentiated NEC component included in the high-grade MiNEN could probably explain the rapid aggravation of the disease. Systemic chemotherapy was applied for the metastatic disease, and the disease was under control with treatment.

The prognosis of gastric MiNEN, especially high-grade, is generally poor. Although early diagnosis and surgical resection of localized disease is crucial to achieve a better prognosis, it is often difficult to diagnose MiNEN with an endoscopic biopsy, before surgical resection. In addition, since the incidence of gastric MiNEN is extremely low, it is not easy to suspect this disease initially. Our case shows that if unexpected clinical deterioration such as liver metastases is observed even after curative resection of gastric cancer, gastric MiNEN should be considered as a differential diagnosis.

\section{CONFLICTS OF INTEREST}

No potential conflict of interest relevant to this article was reported.

\section{ORCID}

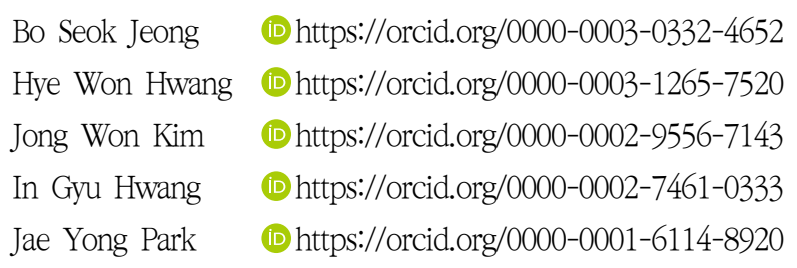

\section{REFERENCES}

1. Cordier R. Argentaffin cells in intestinal tumors. Arch Int Med Exp 1924;1:5.

2. Nagtegaal ID, Odze RD, Klimstra D, et al. The 2019 WHO classification of tumours of the digestive system. Histopathology 2020;76:182-188.

3. Fléjou JF. WHO classification of digestive tumors: the fourth edition. Ann Pathol 2011;31(5 Suppl):S27-S31.

4. Wang J, He A, Feng Q, et al. Gastrointestinal mixed adenoneuroendocrine carcinoma: a population level analysis of epidemiological trends. J Transl Med 2020;18:128.

5. Takahashi K, Fujiya M, Sasaki T, et al. Endoscopic findings of gastric mixed adenoneuroendocrine carcinoma: a case report. Medicine (Baltimore) 2020;99:e22306.

6. Lee HH, Jung CK, Jung ES, Song KY, Jeon HM, Park CH. Mixed exocrine and endocrine carcinoma in the stomach: a case report. J Gastric Cancer 2011;11:122-125.

7. de Mestier L, Cros J. Digestive system mixed neuroendocrinenon-neuroendocrine neoplasms (MiNEN). Ann Endocrinol (Paris) 2019;80:172-173.

8. Frizziero M, Chakrabarty B, Nagy B, et al. Mixed neuroendocrine non-neuroendocrine neoplasms: a systematic review of a controversial and underestimated diagnosis. J Clin Med 2020;9:273.

9. Lee EJ, Park SM, Maeng L, Lee A, Kim KM. Composite glandular-endocrine cell carcinomas of the stomach: clinicopathologic and methylation study. APMIS 2005;113:569-576.

10. La Rosa S, Marando A, Sessa F, Capella C. Mixed adenoneuroendocrine carcinomas (MANECs) of the gastrointestinal tract: an update. Cancers (Basel) 2012;4:11-30.

11. La Rosa S, Sessa F, Uccella S. Mixed neuroendocrine-nonneuroendocrine neoplasms (MiNENs): unifying the concept of a heterogeneous group of neoplasms. Endocr Pathol 2016;27: 284-311.

12. Wu C, Bao W, Rao Q, et al. Clinicopathological features and prognosis of gastric mixed adenoneuroendocrine carcinoma. 
Int J Clin Exp Pathol 2018;11:1499-1509.

13. Kim JJ, Kim JY, Hur H, Cho YK, Han SU. Clinicopathologic significance of gastric adenocarcinoma with neuroendocrine features. J Gastric Cancer 2011;11:195-199.

14. Gurzu S, Kadar Z, Bara T, et al. Mixed adenoneuroendocrine carcinoma of gastrointestinal tract: report of two cases. World J Gastroenterol 2015;21:1329-1333.

15. Wang YH, Lin Y, Xue L, Wang JH, Chen MH, Chen J. Relationship between clinical characteristics and survival of gastroenteropancreatic neuroendocrine neoplasms: a single-institution analysis (1995-2012) in South China. BMC Endocr Disord 2012; 12:30.

16. de Mestier L, Cros J, Neuzillet C, et al. Digestive system mixed neuroendocrine-non-neuroendocrine neoplasms. Neuroendocrinology 2017;105:412-425.

17. Düzköylü Y, Aras O, Bostancı EB, Keklik Temuçin T, Ulaş M. Mixed adeno-neuroendocrine carcinoma; case series of ten patients with review of the literature. Balkan Med J 2018;35: 263-267.

18. Ramos MFKP, Pereira MA, Arabi AYM, et al. Gastric mixed neuroendocrine non-neuroendocrine neoplasms: a western center case series. Med Sci (Basel) 2021;9:47.

19. Lee JH, Kim HW, Kang DH, Choi CW, Park SB, Kim SH. A gastric composite tumor with an adenocarcinoma and a neuroendocrine carcinoma: a case report. Clin Endosc 2013;46:280-283. 